\title{
REVIEW \\ Passive and active roles of fat-free mass in the control of energy intake and body composition regulation
}

\author{
AG Dulloo, J Jacquet, JL Miles-Chan and Y Schutz
}

While putative feedback signals arising from adipose tissue are commonly assumed to provide the molecular links between the body's long-term energy requirements and energy intake, the available evidence suggests that the lean body or fat-free mass (FFM) also plays a role in the drive to eat. A distinction must, however, be made between a 'passive' role of FFM in driving energy intake, which is likely to be mediated by 'energy-sensing' mechanisms that translate FFM-induced energy requirements to energy intake, and a more 'active' role of FFM in the drive to eat through feedback signaling between FFM deficit and energy intake.

Consequently, a loss of FFM that results from dieting or sedentarity should be viewed as a risk factor for weight regain and increased fatness not only because of the impact of the FFM deficit in lowering the maintenance energy requirement but also because of the body's attempt to restore FFM by overeating - a phenomenon referred to as 'collateral fattening'. A better understanding of these passive and active roles of FFM in the control of energy intake will necessitate the elucidation of peripheral signals and energy-sensing mechanisms that drive hunger and appetite, with implications for both obesity prevention and its management.

European Journal of Clinical Nutrition (2017) 71, 353-357; doi:10.1038/ejcn.2016.256; published online 14 December 2016

\section{INTRODUCTION}

Some 30 years ago, Gilbert Forbes pointed out that the lean body mass and body fat are in a sense companions, so that in many situations a change in one is accompanied by a change in the other, and usually in the same direction. ${ }^{1}$ Until recently, however, the extent to which the lean body or fat-free mass (FFM) component in this companionship influences energy intake has been largely ignored, amid a dominant adipocentric view of appetite control that has been reinforced by the discovery of leptin and its relationship with fat mass (FM).

In fact, in an early investigation about the relationships between body composition and objectively measured ad libitum food intake over several weeks in obese and non-obese women, Lissner et al. ${ }^{2}$ showed that the energy intake for the maintenance of body weight was not correlated with adiposity expressed as percent body fat or as FM, but was positively associated with FFM. These findings were ignored or overlooked for the next 25 years despite the more than 100 citations that this publication received, albeit in relation to its other important findings about under-reporting of food intake by both obese and non-obese individuals. It is only recently that the predictive power of FFM on energy intake has gained attention following the demonstration by Blundell et al. ${ }^{3}$ that FFM, but not FM, was positively associated with self-selected meal size and total energy intake in overweight and obese subjects. Further confirmation of a positive association between energy intake with FFM, but not FM, can be derived from studies in adults ${ }^{4-6}$ as well as in overweight and obese adolescents. ${ }^{7}$

Taken together, these findings have strengthened the argument of Lissner et al. ${ }^{2}$ that 'research that focuses on the relationship between energy intake and obesity is misplaced because energy requirement appears to be a direct function of lean mass rather than of adiposity'. Indeed, the predictive power of FFM on energy intake provides one explanation as to why people with obesity, on average, tend to eat more than lean people, given that the development of obesity is accompanied not only by a large gain in FM but also by a gain in FFM, the composition of excess weight gain being $70-80 \%$ fat and $20-30 \%$ $\mathrm{FFM}^{8}$ (Figure 1). In the analysis of the relationship between energy intake and FFM, however, a distinction must be made between (i) a relatively 'passive' role of FFM in driving energy intake, which is likely to be mediated by 'energy-sensing' mechanisms that translate FFM-induced energy requirements to energy intake and (ii) a more 'active' role of FFM in the drive to eat through feedback signaling between FFM deficit and energy intake. These relationships are depicted in Figure 2, and discussed below.

\section{PASSIVE ROLE OF FFM IN DRIVING ENERGY INTAKE}

The effect of a higher FFM associated with obesity on energy intake is expected, given the high metabolic activity of organs and tissues that constitute the FFM-the most important determinant of energy expenditure (EE) and which accounts for $\sim 70 \%$ of the variance in resting EE. ${ }^{9}$ The latter, in turn, is the major determinant of daily EE particularly in the typical sedentary person. Indeed, by applying various statistical models to data on body composition, EE and energy intake (including a mediation model using path analysis approaches), a number of recent studies have shown that the impact of FFM on food intake is not direct, but rather indirect since FFM influences daily hunger, self-selected meals size and daily energy intake via its effect on resting EE. ${ }^{4,6,10}$ It has been proposed by Hopkins and Blundell ${ }^{11}$ that such FFM-induced energy requirements represent 


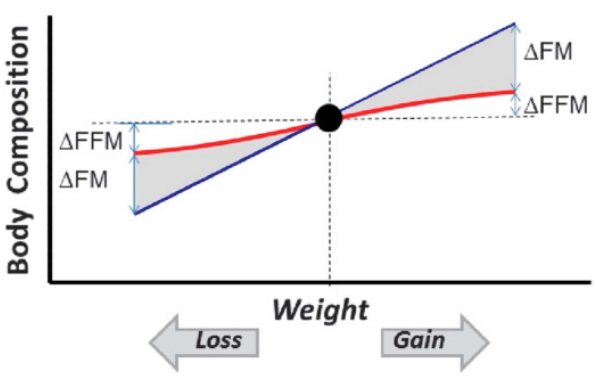

Figure 1. Schematic diagram depicting, in a normal-weight individual, the impact of changes in body weight on body composition. If the individual enters into a chronic positive energy imbalance, the resulting weight gain will comprise not only of an increase in fat but also in FFM: the proportion of excess weight gain as FFM being $20-30 \%,{ }^{8}$ but is known to show large inter-individual variability. Conversely, if the individual enters into a chronic negative energy imbalance, the resulting weight loss will comprise not only of a loss of FM but also in FFM: the proportion of weight loss as FFM being highly dependent upon the initial body fat $\%$ even in the normal range of body mass index. ${ }^{24}$

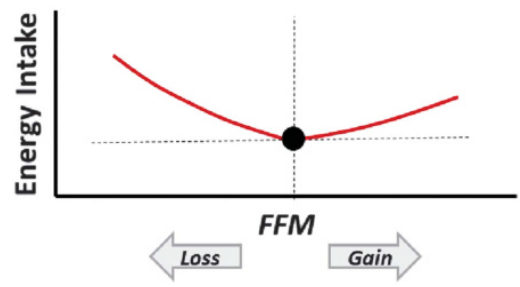

Figure 2. Schematic diagram depicting, in a normal-weight individual, the impact of changes in fat-free mass (FFM) on energy intake. If this individual enters into a chronic positive energy imbalance, the resulting weight gain will comprise not only fat but also FFM $\left(20-30 \%\right.$ of excess weight), ${ }^{8}$ thereby resulting in an increase in resting metabolic rate and hence in an increase in energy needs. A new steady state of body weight (albeit higher than before) is reached when the increase in energy needs matches the positive energy imbalance. Thus, the resulting higher body weight (and higher fat and FFM) is the price to pay to fully offset the initial positive energy imbalance, and at the new steady-state body weight, the energy intake of this individual with obesity would be higher than before the positive energy imbalance occurred, in large part because of the higher FFM and higher energy needs. This can be referred to as a 'passive' influence of FFM on energy intake. By contrast, a more 'active' role of FFM on energy intake is postulated to occur if the normal-weight individual loses FFM. In this case, the deficit in FFM will trigger a feedback signaling pathway arising from FFM to increase energy intake in an attempt to restore FFM, as depicted in Figure 4.

'a physiological source of hunger that drives food intake at a level proportional to basal energy requirements', and that 'this longterm (tonic) signal of energy requirements would help 'tune' energy intake to $E E$, and help ensure the maintenance and execution of key biological and behavioral processes'.

Little is known, however, as to how the body's energy needs are sensed, integrated and translated into eating behaviors. In this context, a recent investigation of how energy needs arising from FFM could be detected by the brain was conducted by Weise et al. ${ }^{12}$ using positron emission tomographic measurement of regional cerebral blood flow in healthy adults with a wide range of adiposity. They reported that FFM, but not FM, was associated with several brain regions involved in the homeostatic control of appetite. While these findings of a link between FFM, hunger and midbrain blood flow may suggest a centrally mediated mechanism whereby EE directly drives FFM-induced energy intake, there is also fragmentary evidence suggesting that energy intake is regulated by one or more molecular signals arising from the FFM or from specific organ masses, in particular the skeletal muscle. This notion, which has formed the basis of an 'aminostatic' theory of appetite control ${ }^{13,14}$ and a 'protein-static' control of food intake in relation to the impetus for lean tissue growth or maintenance, ${ }^{15}$ has particular relevance when considering the dynamic phase of body weight recovery during refeeding or obesity relapse, and hence when examining the relationship between deficits in FFM and increased energy intake (Figure 2).

\section{ACTIVE ROLE OF FFM DEFICIT IN DRIVING ENERGY INTAKE}

A re-analysis of data on food intake and body composition in response to semistarvation and refeeding in the men participating in the classic Minnesota Experiment ${ }^{16}$ has revealed that the FFM deficit, independently of the FM deficit, predicted the degree of hyperphagia that occurred during post-starvation ad libitum refeeding ${ }^{17}$ (Table 1). Furthermore, an examination of the dynamics of regain in FM and FFM during weight recovery revealed that despite the complete recovery of body weight and FM, hyperphagia persisted until FFM were completely restored to baseline (pre-starvation) levels ${ }^{17}$ (Figure 3). Thus, the compensatory hyperphagia as observed in response to energy deprivation and loss of body weight cannot be explained by the lipostatic (or adipostatic) theory alone, and the contribution of the parallel reduction in FFM to the hyperphagic response also needs to be considered. One can therefore postulate the existence of a control system that responds to a loss or deficit in FFM through compensatory increases in energy intake in an attempt to restore FFM, with consequential accompanying increases in FM. Such 'collateral fattening' (Figure 4) has several implications for research in energy balance in general, and more specifically in both the prevention and treatment of obesity.

Impact of FFM loss in countering efficacy of obesity therapy

Loss in FFM accompanying diet-induced weight reduction is a factor that contributes to weight regain in terms of a lowering in the energy cost of FFM maintenance (and consequently reduced resting $\mathrm{EE})$, but also, as discussed above, in terms of the stimulatory effect of FFM loss on appetite. In this context, it may have relevance to the current debate about whether rapid weight loss by very-low calorie diets, by virtue of a larger energy deficit and lower absolute amount of protein, compared with a more gradual weight loss approach by low calorie diets, increases the risk for greater FFM loss and greater susceptibility to weight regain. ${ }^{18}$ Furthermore, there is evidence that the amount of sleep plays an important role in the preservation of FFM during periods of reduced caloric intake, such that lack of sufficient sleep may compromise the efficacy of typical dietary interventions for weight loss. In a randomized crossover study, ${ }^{19}$ exposure of overweight middle-aged adults to 2 weeks of a reduced calorie diet combined with sleep restriction ( 5.5 vs $8.5 \mathrm{~h}$ of night time sleep opportunity) produced a catabolic state characterized by a significant decrease in the loss of FM (1.4 vs $0.6 \mathrm{~kg}$ ) and considerably increased loss of FFM (1.5 vs $2.4 \mathrm{~kg} ; P<0.01)$. These greater losses in FFM were accompanied not only by changes in EE and a shift in relative substrate utilization towards diminished fat oxidation, but also by increased hunger. ${ }^{19}$

Fat overshooting in response to dieting and weight cycling The existence of a control system operating through a negative feedback loop between FFM and energy intake provides a 
Table 1. Predictors of the total hyperphagic response (integrated hyperphagia) in men refed ad libitum in the Minnesota Experiment

\begin{tabular}{lc}
\hline Independent variables & $r^{2}$ \\
\hline Fat mass to be recovered (step 1) & 0.32 \\
Prior energy intake deficit (step 2) & 0.68 \\
Fat-free mass to be recovered (step 3) & 0.80 \\
\hline
\end{tabular}

The results are obtained by stepwise regression analysis of integrated hyperphagia vs the following independent variables: fat mass to be recovered, fat-free mass to be recovered and prior energy intake deficit. Adapted from Dulloo et al. ${ }^{17}$

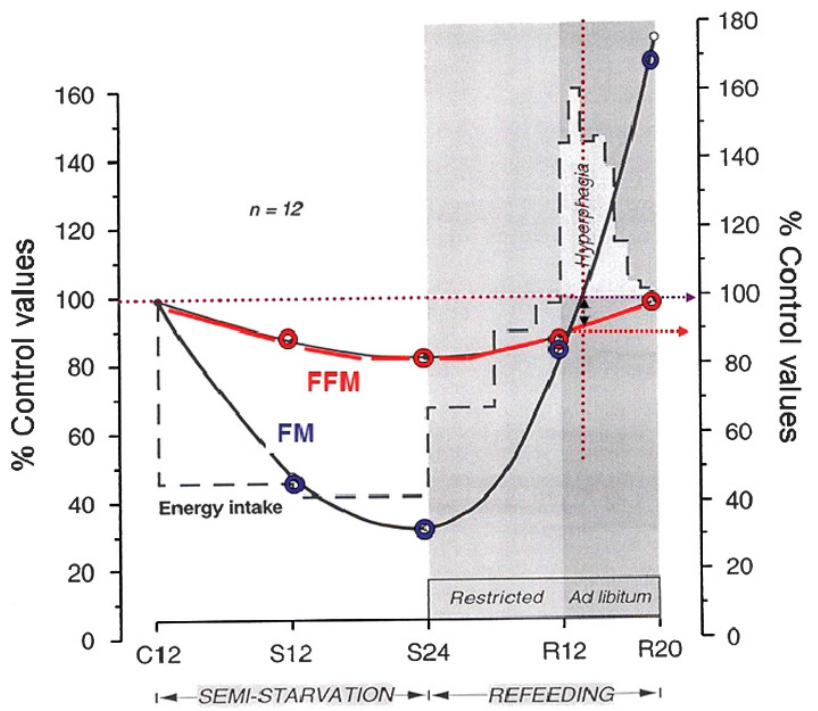

Figure 3. Dynamics of body composition changes in men participating in all phases of the Minnesota Experiment. The data are plotted to show the pattern of changes in energy intake, body fat mass (FM) and fat-free mass (FFM) during semistarvation and refeeding in the 12 men who completed all phases of the Minnesota Experiment (including the ad libitum phase of refeeding). All values are expressed as percentages of corresponding values during the control (pre-starvation) period. C12: end of 12 weeks of control period; S12 and S24: end of 12 and 24 weeks of semistarvation respectively; R12 and R20: end of 12 weeks of restricted refeeding and 8 weeks of ad libitum refeeding, respectively. The doubleheaded arrow indicates that at the time-point when body fat had been fully recovered (that is, $100 \%$ of control period value), FFM recovery is still far from complete, with hyperphagia persisting until completion of FFM recovery. Adapted from Dulloo et al. ${ }^{17}$

mechanistic explanation as to why dieting and weight cycling confer increased risk for future weight gain in those who are of normal body weight (but perceived themselves to be fatter than they really are) rather than in those who are actually obese. ${ }^{20}$ Indeed, the phenomenon of 'fat overshooting', whereby the amount of FM regained is in excess of the amount of FM lost, has been reported in normal-weight adults following weight recovery from semistarvation in the Minnesota Experiment ${ }^{16}$ or from training-induced weight loss of US army Rangers in a multistressor environment that includes energy deficit, ${ }^{21-23}$ the excess body fat being $4-5 \mathrm{~kg}$ on average. The explanation for this phenomenon of fat overshooting resides in the fact that (i) the proportion of weight loss as FFM is higher in lean individuals than obese $^{24}$ (Figure 5a) and that (ii) following substantial weight loss, lean individuals regain FM at a higher rate than FFM (termed

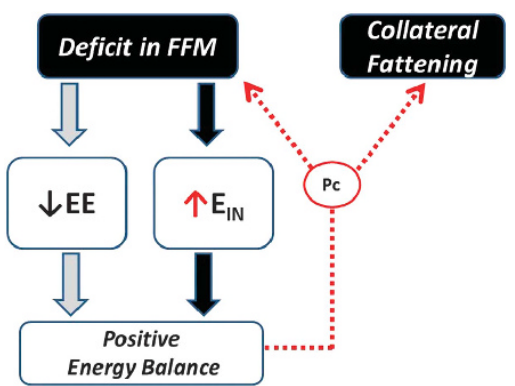

Figure 4. Concept of 'Collateral Fattening'. A deficit in FFM results not only in a lower energy expenditure (EE) and hence lower energy needs for weight maintenance, but also in the activation of a feedback loop that drives energy intake $\left(E_{\mathrm{IN}}\right)$ in an attempt to restore FFM through the lean-to-fat partitioning characteristic (Pc) of the individual.

'preferential catch-up fat'): this asymmetry in FM and FFM recovery having been observed in studies of recovery from experimental semistarvation ${ }^{16}$ or more moderate caloric restriction, ${ }^{25}$ as well as during weight recovery in famine victims and in patients recovering from disease cachexia and anorexia nervosa (see Dulloo $^{26}$ for a review). It is to be noted that a recent study by Müller et al. $^{27}$ did not show preferential catch-up fat during refeeding after 3 weeks of caloric restriction, most probably because the degree of fat depletion over this period was rather mild-only $\sim 6 \%$ relative to baseline 'habitual' levels. This contrasts with the earlier studies mentioned above, $16,21-23,25,26$ where the degree of fat depletion was greater than $10 \%$ of initial values. It is therefore likely that some kind of 'threshold' level of fat depletion needs to be exceeded in order for the phenomena of preferential catch-up fat and fat overshooting to occur.

This misalignment in the time-course of FM and FFM replenishment results in an increased drive to eat which persists beyond the point by which FM is restored, ${ }^{16,23}$ until complete FFM recovery. As such the excess fat accumulated during this last phase of FFM restoration (fat overshooting) is a requirement in achieving complete recovery of FFM, thereby underlying 'collateral fattening' (Figure 4). The re-analysis of data collected during the Minnesota Experiment also provides evidence to suggest that non-obese dieters are more at-risk for fat overshooting than individuals who are obese at the start of dietary restriction, by showing an exponential increase in the extent of fat overshoot with decreasing pre-starvation $\%$ body fat. ${ }^{20}$ This relationship, shown in Figure $5 \mathrm{~b}$ provides proof-of-concept that non-obese dieters are at greater risk for fat overshooting than the obese dieters. This is of particular relevance in light of the increasing prevalence of dieting among normal-weight individuals ${ }^{28}$ and the weight cycling that occurs through repeated dieting; both of which have the potential to provide a pathway to obesity in otherwise normal-weight individuals, and thus of concern in terms of public health.

Sedentariness, FFM deficit and predisposition to obesity While body weight depends ultimately on the balance between energy entering the body as food and energy expended, an increase in physical activity may affect energy balance other than simply increasing EE through time spent in physical work. A frequently quoted study on the relationship between physical activity and body fat content is that by Mayer et al., ${ }^{29}$ who examined a group of workers in a jute factory in India in the mid1950 s and found that above a certain critical level of physical activity, food intake increased in line with work load and no change in body weight occurred. However, at very-low physical activity levels, the relationship broke down and both food intake 


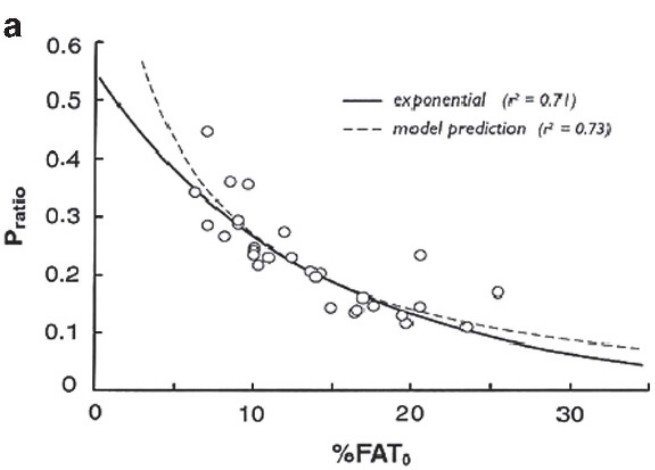

b

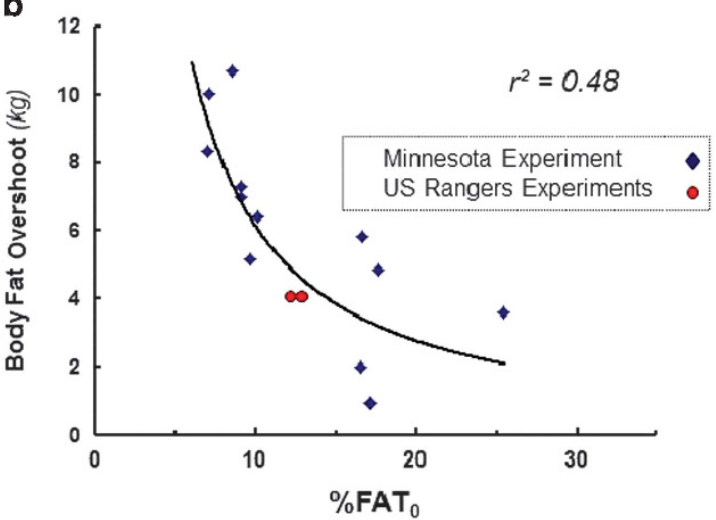

Figure 5. (a) Relationship between the proportions of energy mobilized as protein (P-ratio) during weight loss due to semistarvation and the initial percentage body fat (\%FAT 0 ) in men of the Minnesota Experiment. Note that P-ratio (expressed in energy terms) is a proxy of the fraction of weight loss as FFM (that is, $\Delta$ FFM/ $\Delta$ Weight). Adapted from Dulloo and Jacquet. ${ }^{24}$ (b) Relationship between the extent of fat overshooting (kg excess fat regained) and the initial (pre-starvation) percentage body fat (\%FAT $)$. The exponential curve is drawn from data on the 12 men who participated in all phases of the Minnesota experiment (data for each individual is represented by a blue-filled diamond symbol). The mean values for men $(n=10)$ participating in each of the Army Ranger training experiments for which body composition data are available $2^{21,23}$ are shown as red-filled circles. Adapted from Dulloo et al. ${ }^{20}$

and body weight increased. In keeping with these early findings of Mayer et $\mathrm{al}_{1}^{29}$ there is increasing support of a J-shaped relationship between levels of physical activity and energy intake, ${ }^{30}$ with intake tightly coupled to expenditure at moderate to high activity levels (thereby promoting the maintenance of energy balance and body fat), but dysregulation occurring at lower activity levels, whereby sedentariness increases with no compensatory decrease in energy intake and an increase in adiposity. With increasing sedentariness, decreased muscle usage and contractile function will likely lead to subsequent loss of FFM-a contention that is supported by the demonstration that even short ( $<10$ days) periods of muscle disuse induced by strict bed rest in healthy young men resulted in diminished skeletal muscle size and substantial loss of lean body mass. $^{31}$ In turn, such FFM loss may stimulate a compensatory increase in energy intake so as to promote the restoration of lean tissue (whether successful or not), with a concomitant increase in FM (Figure 4).

\section{Search for protein-stats}

While evidence of a direct relationship between FFM deficit and energy intake mounts, the molecular signaling mechanisms underlying this relationship, including the specific trigger source (whole-body protein mass, skeletal muscle mass, other organ size or metabolic activity), remains elusive. However, with skeletal muscle myocytes now known to secrete several hundred factors, ${ }^{32}$ the search for FFM-derived/sensitive feedback signals ('proteinstats') to the central appetite regulatory centers has begun. In this context, appropriate models of FFM manipulation must be developed. The laboratory of Berthoud may well have developed one such model, with diet-induced obese mice that underwent a post-caloric restriction Roux-en-Y gastric bypass showing higher energy intake and weight regain in the post-surgical period, but with the weight regained comprising exclusively of FFM. ${ }^{33}$ Thus, by remaining sensitive to changes in lean mass, but eliminating the defense of fat mass, this mouse model provides an interesting opportunity to elucidate the feedback signals from FFM that drive energy intake, with major implications for the pathogenesis and management of obesity and cachexia.

\section{CONCLUDING REMARKS}

Although putative feedback signals arising from adipose tissue (for example, leptin) are commonly assumed to provide the molecular link between long-term energy needs and daily energy intake, the available evidence suggests that the lean body mass also plays a role in the drive to eat and in achieving energy balance. In addressing the role of FFM in the drive on hunger, appetite or satiety, however, it is important to distinguish between (i) a relatively 'passive' role of FFM on energy intake which is mediated by 'energy-sensing' mechanisms that translate FFMinduced energy needs to energy intake and (ii) a more 'active' role of FFM in the drive to eat through feedback signals between FFM deficit and energy intake. Thus, the loss of FFM resulting from dieting or sedentarity should not be viewed solely as contributing to weight regain and fatness because of the consequentially lower maintenance EE of a lower FFM, but also to the body's attempt to restore FFM by overeating. From an evolutionary standpoint, this active role of FFM in driving hunger/appetite to restore its mass and hence functional capacity after FFM loss (which is potentially life-threatening) can be regarded as adaptive. However, the accompanying, 'collateral fattening' may be of particular consequence in situations where the body's capacity to rebuild lean tissue is impaired-such as during aging and malnutrition. The degree to which this fattening occurs under these conditions is therefore of interest, as is also the extent to which this process may operate under conditions of sarcopenic obesity. Regardless, while peripheral signals and energy-sensing mechanisms remain to be elucidated, a better understanding of the roles of FFM (both active and passive) in the control of energy intake, hunger and appetite is of importance to both the maintenance and restoration of FFM, with implications for obesity prevention and management.

\section{CONFLICT OF INTEREST}

The authors declare no conflict of interest.

\section{ACKNOWLEDGEMENTS}

This research work was supported by the Swiss National Science Foundation (grant no. 310030-152870)

\section{REFERENCES}

1 Forbes GB. Lean body mass-body fat interrelationships in humans. Nutr Rev 1987; 45: 225-231.

2 Lissner L, Habicht JP, Strupp BJ, Levitsky DA, Haas JD, Roe DA. Body composition and energy intake: do overweight women overeat and underreport? Am J Clin Nutr 1989; 49: 320-352.

3 Blundell JE, Caudwell P, Gibbons C, Hopkins M, Näslund E, King NA et al. Body composition and appetite: fat-free mass (but not fat mass or BMI) is positively 
associated with self-determined meal size and daily energy intake in humans. Br J Nutr 2012; 107: 445-449.

4 Caudwell P, Finlayson G, Gibbons C, Hopkins M, King N, Näslund E et al. Resting metabolic rate is associated with hunger, self-determined meal size, and daily energy intake and may represent a marker for appetite. Am J Clin Nutr 2013; 97: 7-14.

5 Weise CM, Hohenadel MG, Krakoff J, Votruba SB. Body composition and energy expenditure predict ad-libitum food and macronutrient intake in humans. Int $J$ Obes (Lond) 2014; 38: 243-251.

6 Piaggi P, Thearle MS, Krakoff J, Votruba SB. Higher daily energy expenditure and respiratory quotient, rather than fat-free mass, independently determine greater ad libitum overeating. J Clin Endocrinol Metab 2015; 100: 3011-3020.

7 Cameron JD, Sigal RJ, Kenny GP, Alberga AS, Prud'homme D, Phillips P et al. Body composition and energy intake-skeletal muscle mass is the strongest predictor of food intake in obese adolescents: The HEARTY trial. Appl Physiol Nutr Metab 2016; 41: 611-617.

8 Webster JD, Hesp R, Garrow JS. The composition of excess weight in obese women estimated by body density, total body water and total body potassium. Hum Nutr Clin Nutr 1984; 38: 299-306.

9 Ravussin E, Burnand B, Schutz Y, Jéquier E. Twenty-four-hour energy expenditure and resting metabolic rate in obese, moderately obese, and control subjects. Am J Clin Nutr 1982; 35: 566-573.

10 Hopkins M, Finlayson G, Duarte C, Whybrow S, Ritz P, Horgan GW et al. Modelling the associations between fat-free mass, resting metabolic rate and energy intake in the context of total energy balance. Int J Obes (Lond) 2016; 40: 312-318.

11 Hopkins M, Blundell JE. Energy balance, body composition, sedentariness and appetite regulation: pathways to obesity. Clin Sci (Lond) 2016; 130: 1615-1628.

12 Weise CM, Thiyyagura P, Reiman EM, Chen K, Krakoff J. A potential role for the midbrain in integrating fat-free mass determined energy needs: An H2 (15) O PET study. Hum Brain Mapp 2015; 36: 2406-2415.

13 Mellinkoff SM, Franklund M, Bouyle D, Greipel M. Relationships between serum amino acid concentration and fluctuations in appetite. J Appl Physiol 1956; 8: 535-538.

14 Bray GA. Amino acids, protein and body weight. Obes Res 1997; 5: 373-379.

15 Millward DJ. A protein-stat mechanism for the regulation of growth and maintenance of the lean body mass. Nutr Res Rev 1995; 8: 93-120.

16 Keys A, Brozek J, Henschel A, Mickelsen O, Taylor HL. The Biology of Human Starvation. University of Minnesota Press: Minnesota, 1950.

17 Dulloo AG, Jacquet J, Girardier L. Poststarvation hyperphagia and body fat overshooting in humans: a role for feedback signals from lean and fat tissues. $A m$ J Clin Nutr 1997; 65: 717-723.

18 Vink RG, Roumans NJ, Arkenbosch LA, Mariman EC, van Baak MA. The effect of rate of weight loss on long-term weight regain in adults with overweight and obesity. Obesity (Silver Spring) 2016; 24: 321-327.

19 Nedeltcheva AV, Kilkus JM, Imperial J, Schoeller DA, Penev PD. Insufficient sleep undermines dietary efforts to reduce adiposity. Ann Intern Med 2010; 153: 435-441.

20 Dulloo AG, Jacquet J, Montani JP, Schutz Y. How dieting makes the lean fatter: from a perspective of body composition autoregulation through adipostats and proteinstats awaiting discovery. Obes Rev 2015; 16(Suppl 1): 25-35.
21 Nindl BC, Friedl KE, Frykman PN, Marchitelli LJ, Shippee RL, Patton JF. Physical performance and metabolic recovery among lean, healthy men following a prolonged energy deficit. Int J Sports Med 1997; 18: 317-3241997.

22 Young AJ, Castellani JW, O'Brien C, Shippee RL, Tikuisis P, Meyer LG et al. Exertional fatigue, sleep loss, and negative energy balance increase susceptibility to hypothermia. J Appl Physiol 1998; 85: 1210-1217.

23 Friedl KE, Moore RJ, Hoyt RW, Marchitelli LJ, Martinez-Lopez LE et al. Endocrine markers of semistarvation in healthy lean men in a multistressor environment. J Appl Physiol 2000; 88: 1820-1830.

24 Dulloo AG, Jacquet J. The control of partitioning between protein and fat during human starvation: its internal determinants and biological significance. Br J Nutr 1999; 82: 339-356.

25 Weyer C, Walford RL, Harper IT, Milner M, MacCallum T, Tataranni PA et al. Energy metabolism after 2 y of energy restriction: the biosphere 2 experiment. Am J Clin Nutr 2000; 72: 946-953.

26 Dulloo AG, Jacquet J, Seydoux J, Montani JP. The thrifty 'catch-up fat' phenotype: its impact on insulin sensitivity during growth trajectories to obesity and metabolic syndrome. Int J Obes (Lond) 2006; 30(Suppl 4): S23-S35.

27 Müller MJ, Enderle J, Pourhassan M, Braun W, Eggeling B, Lagerpusch M et al. Metabolic adaptation to caloric restriction and subsequent refeeding: the Minnesota Starvation Experiment revisited. Am J Clin Nutr 2015; 102: 807-819.

28 Montani JP, Schutz Y, Dulloo AG. Dieting and weight cycling as risk factors for cardiometabolic diseases: who is really at risk? Obes Rev 2015; 16(Suppl 1): 7-18.

29 Mayer J, Roy P, Mitra KP. Relation between caloric intake, body weight, and physical work: studies in an industrial male population in West Bengal. Am J Clin Nutr 1956; 4: 169-175.

30 Shook RP, Hand GA, Drenowatz C, Hebert JR, Paluch AE, Blundell JE et al. Low levels of physical activity are associated with dysregulation of energy intake and fat mass gain over 1 year. Am J Clin Nutr 2015; 102: 1332-1338.

31 Dirks ML, Wall BT, van de Valk B, Holloway TM, Holloway GP, Chabowski A et al. One week of bed rest leads to substantial muscle atrophy and induces wholebody insulin resistance in the absence of skeletal muscle lipid accumulation. Diabetes 2016; 65: 2862-2875.

32 Pedersen BK, Febbraio MA. Muscles, exercise and obesity: skeletal muscle as a secretory organ. Nat Rev Endocrinol 2012; 8: 457-465.

33 Hao Z, Mumphrey MB, Townsend RL, Morrison CD, Münzberg H, Ye J et al. Reprogramming of defended body weight after Roux-En-Y gastric bypass surgery in diet-induced obese mice. Obesity (Silver Spring) 2016; 24: 654-660.

(i) $\Theta$ This work is licensed under a Creative Commons Attributioncc) NonCommercial-NoDerivs 4.0 International License. The images or other third party material in this article are included in the article's Creative Commons license, unless indicated otherwise in the credit line; if the material is not included under the Creative Commons license, users will need to obtain permission from the license holder to reproduce the material. To view a copy of this license, visit http:// creativecommons.org/licenses/by-nc-nd/4.0/

(c) The Author(s) 2017 